EPJ manuscript No.

(will be inserted by the editor)

\title{
Atom interferometry based on light pulses : application to the high precision measurement of the ratio $h / m$ and the determination of the fine structure constant
}

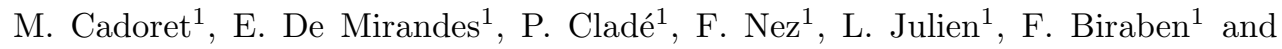 \\ S. Guellati-Khélifa ${ }^{1,2, a}$ \\ ${ }^{1}$ Laboratoire Kastler Brossel, Université Pierre et Marie Curie, ENS, CNRS, 4 place Jussieu, 75252 \\ Paris Cedex 05, France \\ ${ }^{2}$ LNE-INM, Conservatoire National des Arts et Métiers, 61 rue du Landy, 93210 La Plaine Saint \\ Denis, France
}

\begin{abstract}
In this paper we present a short overview of atom interferometry based on light pulses. We discuss different implementations and their applications for high precision measurements. We will focus on the determination of the ratio $h / m$ of the Planck constant to an atomic mass. The measurement of this quantity is performed by combining Bloch oscillations of atoms in a moving optical lattice with a Ramsey-Bordé interferometer.
\end{abstract}

\section{Introduction}

Intense progress in laser cooling has led to the building of reliable atom interferometers. A broad overview of accomplishments in matter wave interferometry was presented by Berman over a decade ago [1]. To implement such an interferometer, optical elements based both on the mechanical forces of light [2|34|5|67] and nanofabricated structures [8 9|10] have been investigated. Right from the outset, light-based atom interferometry became a technique of choice for high precision measurements. This method has benefited greatly from the impressive progress in laser technology. In this lecture we focus on atom interferometers based on light pulses.

${ }^{a}$ e-mail: guellati@spectro.jussieu.fr 
In such an interferometer we consider the atomic interference of internal states. The spatial separation of the atoms is produced by the momentum recoil induced by the electromagnetic field used to drive the atoms from one internal state to another. In this way, the internal and external degrees of freedom are strongly linked. Conceptually, such an interferometer provides a measurement of the recoil frequency. This lecture is divided in two main parts. The first is devoted to the analysis of atom interferometer based on light pulses. After a description of the basic concepts such as Rabi oscillations and stimulated Raman transitions, we use a simple formalism based on a plane wave to describe Ramsey-Bordé interferometers. We discuss different configurations and their application to high-precision measurements. In the second part, we describe an experiment underway in our group at Laboratoire Kastler Brossel which combines Bloch oscillations with a Ramsey Bordé interferometer. Using this approach, we aim to determine the ratio $h / m_{\mathrm{Rb}}$ (where $m_{\mathrm{Rb}}$ is the mass of the rubidium atom) and hence a value for the fine structure constant $\alpha$.

\section{Part I: Atom interferometry based on optical pulses}

\section{Basic concepts}

\subsection{Two-level atom interacting with travelling waves: Rabi oscillations}

Let us consider a two-level atomic system $(|g\rangle$ and $|e\rangle)$ with respective energies $\hbar \omega_{g}$ and $\hbar \omega_{e}$. The levels are coupled by an electromagnetic field of angular frequency $\omega$ and wave vector $\mathbf{k}$. We denote by $\Omega$ the Rabi frequency which represents the coupling constant. In the rotating wave approximation, the Hamiltonian is given by:

$$
H=\frac{\widehat{\mathbf{p}}^{2}}{2 m}+\hbar \omega_{e}|e\rangle\left\langle e\left|+\hbar \omega_{g}\right| g\right\rangle\langle g|+\left[\hbar \Omega e^{i(\mathbf{k} \hat{\mathbf{x}}-\omega t)}|e\rangle\left\langle g\left|+\hbar \Omega^{*} e^{-i(\mathbf{k} \hat{\mathbf{x}}-\omega t)}\right| g\right\rangle\langle e|\right]
$$

where $\widehat{\mathbf{p}}$ operates on the momentum part of the atomic state.

A complete description of the atom-light interaction should include both the internal energy states and the external degrees of freedom. Such a description is necessary to analyze an atom interferometer. One should consider explicitly the propagation of spatial wave packets in order to calculate the phase shifts between interfering paths due both to the free-space propagation and to the atom-light interaction. The usual approach is to consider the atomic wave packets as the sum of plane-wave momentum states. The momentum transfer due to the interaction of the electromagnetic field is calculated for a given plane-wave component, after which the integral 
over all of the momentum states in the atomic ensemble is performed. The atomic states are labeled by $\left|g, \mathbf{p}_{\mathbf{g}}\right\rangle$ or $\left|e, \mathbf{p}_{\mathbf{e}}\right\rangle\left(\left|g, \mathbf{p}_{\mathbf{g}}\right\rangle=|g\rangle \otimes\left|\mathbf{p}_{\mathbf{g}}\right\rangle\right.$ and $\left.\left|e, \mathbf{p}_{\mathbf{e}}\right\rangle=|e\rangle \otimes\left|\mathbf{p}_{\mathbf{e}}\right\rangle\right)$. In the momentum basis, the spatial dependence arises via the translation operator $e^{\mathbf{k} \cdot \hat{\mathbf{x}}}$ :

$$
e^{i \mathbf{k} \cdot \hat{\mathbf{x}}}|\mathbf{p}\rangle=|\mathbf{p}+\mathbf{h} \mathbf{k}\rangle
$$

This equation expresses the well-known result whereby the absorption of a photon of wave vector $\mathbf{k}$ changes the atomic momentum by $\hbar \mathbf{k}$. The light field couples the quantum states $|g, \mathbf{p}\rangle$ and $|e, \mathbf{p}+\mathbf{h} \mathbf{k}\rangle$.

For simplicity, we use the transformations $|\tilde{g}\rangle=,e^{i \omega_{g} t}|g\rangle$ and $|\tilde{e}\rangle=e^{i \omega_{e} t}|e\rangle$. The hamiltonian becomes:

$$
H=\frac{\widehat{\mathbf{p}}^{2}}{2 m}+\left[\hbar \Omega e^{i(\mathbf{k} \hat{x}-\phi(t))}|\tilde{e}\rangle\left\langle\tilde{g}\left|+\hbar \Omega^{*} e^{-i(\mathbf{k} \hat{x}-\phi(t))}\right| \tilde{g}\right\rangle\langle\tilde{e}|\right]
$$

where the phase $\phi(t)=\delta t$, with detuning $\delta=\omega-\left(\omega_{e}-\omega_{g}\right)$. This hamiltonian describes the transition from $|g\rangle$ to $|e\rangle$ associated with the transfer of photon momentum $\hbar \mathbf{k}$.

The time evolution of the quantum state $|\psi(t)\rangle$ can be expressed in terms of the timedependent coefficients $a_{g, \mathbf{p}}(t)$ and $a_{e, \mathbf{p}+\hbar \mathbf{k}}(t)$ :

$$
|\Psi(t)\rangle=a_{e, \mathbf{p}+\hbar \mathbf{k}}(t)|\tilde{e}, \mathbf{p}+\hbar \mathbf{k}\rangle e^{-i \frac{|\mathbf{p}+\hbar \mathbf{k}|^{2}}{2 m \hbar} t}+a_{g, \mathbf{p}}(t)|\tilde{g}, \mathbf{p}\rangle e^{-i \frac{|\mathbf{p}|^{2}}{2 m \hbar} t}
$$

These coefficients can be calculated by solving the Schrödinger equation :

$$
i \hbar \frac{d}{d t}|\Psi(t)\rangle=H|\Psi(t)\rangle
$$

In the limit where $\phi(t)$ is constant during the pulse, the probability of finding an atom in state $|e\rangle$ after an interaction time $\tau$ is then given by :

$$
\left|a_{e, \mathbf{p}+\hbar \mathbf{k}}(t)\right|^{2}=\frac{1}{2}(1-\cos \Omega \tau)
$$

The atom undergoes the well-known Rabi oscillations between the two states $|g\rangle$ and $|e\rangle$.

Two configurations are relevant for atom interferometry in the particular case where $a_{g}\left(t_{0}\right)=$ 1 and $a_{e}\left(t_{0}\right)=0$ : 
- Case $\boldsymbol{I}: \pi / 2$-light pulse here corresponding to $\Omega \tau=\pi / 2$

$$
|\Psi(t)\rangle=\frac{1}{\sqrt{2}}\left[|\tilde{g}, \mathbf{p}\rangle-i|\tilde{e}, \mathbf{p}+\mathbf{h} \mathbf{k}\rangle e^{-i \phi(t)}\right]
$$

- Case II : $\pi$-light pulse i.e. $\Omega \tau=\pi$

$$
|\Psi(t)\rangle=-i|\tilde{e}, \mathbf{p}+\mathbf{h} \mathbf{k}\rangle
$$

When the atom is subjected to a $\pi / 2$ light pulse, the photon of momentum $\hbar \mathbf{k}$ puts it into a coherent and equal superposition of both energy states. The recoil imparted to the atom gives rise to two coherent wave packets (in each internal state) separated by a velocity $v_{r}=\hbar \mathbf{k} / m$. In others words, this $\pi / 2$ light pulse plays a role analogous to the 50-50 beam splitter in classical optics. In the same way, when the coupling parameters are chosen such that $\Omega_{e g} \tau=\pi$ (a $\pi$ pulse), the probability of finding an atom in $|e, p+\hbar \mathbf{k}\rangle$ after a time $\tau$ is equal to unity; in this case the light pulse behaves like a mirror.

We should emphasize that two terms play an important role for calculating phase shift in atom interferometer: the kinetic energy $p^{2} / 2 m$ (external degree of freedom) and the phase $\phi(t)$. The latter depends both on the laser frequency and on the internal energy of the atoms. In this particular case, we have chosen a fixed laser frequency and constant internal energy for the atom. More generally, to include the situations where the detuning $\delta$ varies through the laser frequency (frequency sweep or phase jump, for example) or through the energy of the atomic levels (i.e. light shift, Zeeman effect), one has to evaluate $\phi(t)=\int \delta(t) \mathrm{d} t$.

Up to now we have neglected spontaneous emission. This omission is valid for the case where the final state $|e\rangle$ is stable enough so that the radiative decay during the sequence pulses is negligible. To implement an atom interferometer based on light pulses the "clock" transitions between the hyperfine levels of an atomic ground state constitute an ideal scheme (for instance the clock transition $\left|F=3, m_{F}=0\right\rangle \longmapsto\left|F=4, m_{F}=0\right\rangle$ in ${ }^{133} \mathrm{Cs}$ or $\left|F=1, m_{F}=0\right\rangle \longmapsto$ $\left|F=2, m_{F}=0\right\rangle$ in $\left.{ }^{87} \mathrm{Rb}\right)$. In addition, these transitions have no first-order Zeeman shifts so that the interferometers are insensitive to the residual magnetic fields. However, since the splitting lies in the microwave range, the recoil imparted by a single microwave photon would be only about $0.1 \mu \mathrm{m} / \mathrm{s}$, making the splitting between the two interfering atomic wavepackets too small to build a sensitive interferometer. Fortunately, one can obtain a large recoil and take 
advantage of the long lifetimes by the use of stimulated two-photon Raman transitions between these same levels [11.

\subsection{Interaction with two counterpropagating waves: stimulated Raman transition}

Let us consider a three level system $(|g\rangle,|e\rangle,|i\rangle)$ where two ground state hyperfine levels $|g\rangle,|e\rangle$ are coupled to an intermediate state $|i\rangle$ by two lasers of angular frequencies $\omega_{1}$ and $\omega_{2}$ and wave vectors $\mathbf{k}_{1}$ and $\mathbf{k}_{2}$ (see Figure. 1). The coupling constants are respectively the Rabi frequencies $\Omega_{1}$ and $\Omega_{2}$.

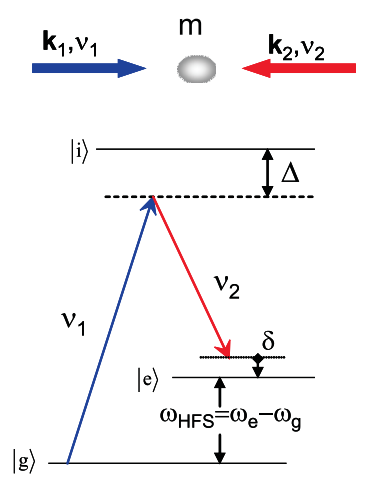

Fig. 1. Energy levels and laser frequencies used for Raman transition.

In the case where the Raman lasers are far-detuned from resonance with $|g\rangle \longrightarrow|i\rangle$ or $|e\rangle \longrightarrow|i\rangle$ single photon transitions $\left(\Delta \gg \Gamma_{i}\right.$ where $\Gamma_{i}$ is the natural linewidth of the level $|i\rangle$ (see Figure 10), the population of the intermediate state $|i\rangle$ remains small and the three level system can be treated as a two-levels system $(|g\rangle,|e\rangle)$ coupled with an effective Rabi frequency $\Omega_{\text {eff }}=\left(\Omega_{1} \Omega_{2}^{*}\right) / 2$. The two-photon Raman excitation can be simply mimed by a single wave of frequency $\omega_{1}-\omega_{2}$ and effective wavevector $\mathbf{k}_{\mathrm{eff}}=\mathbf{k}_{1}-\mathbf{k}_{2}$. This two-levels system will undergo Rabi oscillations at $\Omega_{\text {eff }}$ (see the previous paragraph). We notice first that when the beams are counterpropagating $\left(\mathbf{k}_{1}-\mathbf{k}_{2} \simeq 2 \mathbf{k}_{1}\right)$, the transition has a Doppler sensitivity twice that of a single-photon optical transition. For ${ }^{87} \mathrm{Rb}$ atoms, the imparted recoil $\left(2 v_{r}\right)$ is about $12 \mathrm{~mm} / \mathrm{s}$, about five orders of magnitude larger than that of the corresponding single-photon microwave transition. Second, to implement successfully stimulated Raman transitions, one does not require ultrastable laser frequencies : only the difference $\left(\omega_{1}-\omega_{2}\right)$ needs to be stable regarding to the hyperfine transition. 

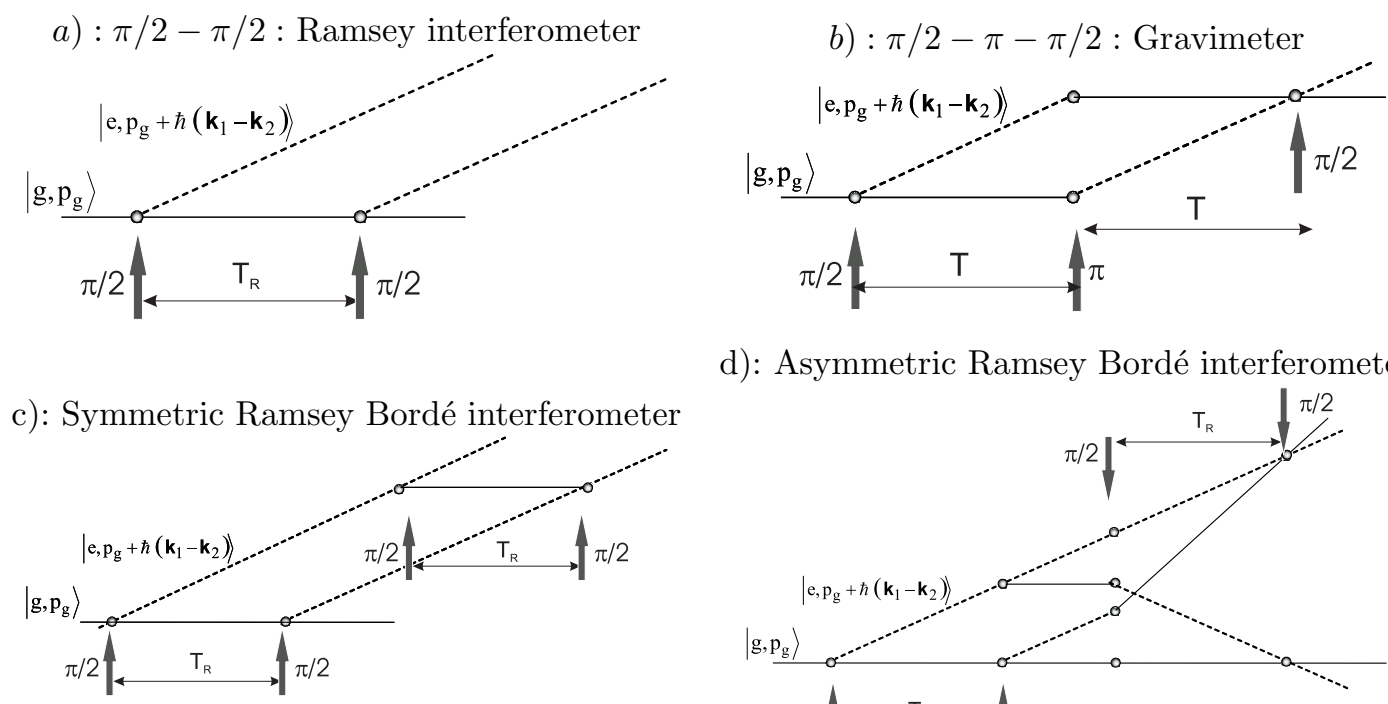

d): Asymmetric Ramsey Bordé interferometer

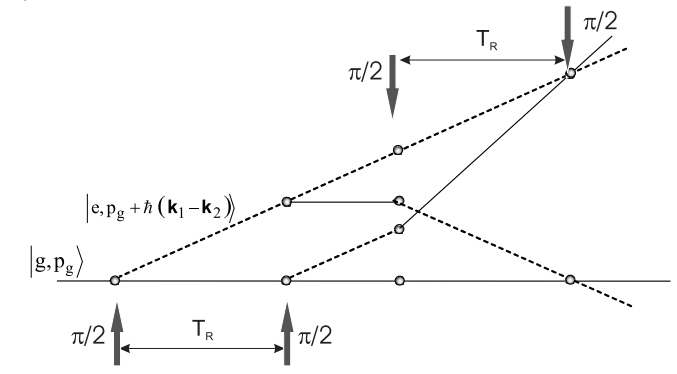

Fig. 2. Recoil diagrams for different configurations of atomic interferometer based on sequences of light pulses (in time-space domain).

\section{Theoretical treatment of the Ramsey-Bordé interferometer}

Atom interferometry based on a light pulses sequence is inspired by Ramsey's separated oscillatory field methods introduced around 1950 to improve the stability of atomic clocks. Thus the most basic scheme of atom interferometer is based on a $\pi / 2-\pi / 2$ pulse sequence. Later, a pulse sequence of two pairs of $\pi / 2$ laser pulses was first proposed by [12]13 to improve the resolution in saturation spectroscopy. The interpretation of four zone Ramsey spectroscopy in terms of atom interferometry with separated wave packets was suggested by Bordé [314]. This scheme has thus become known as a Ramsey-Bordé interferometer.

In this section we analyze such an interferometer. We consider that each light pulse drives a Raman transition between the two hyperfine states $|g\rangle$ and $|e\rangle$. We first calculate the phase shift between interfering paths in the case of a simple $\pi / 2-\pi / 2$ sequence. Then we investigate the different configurations for implementation of the more relevant $\pi / 2-\pi / 2-\pi / 2-\pi / 2$ scheme. The most general and widely used approach to calculate the phase shift is based on the path integral formalism [315]16. However for simplicity, we shall use an approach based on a plane-wave decomposition, which is strictly valid only when the atoms are subjected to uniform forces. This is indeed the case for the applications we describe. 


\subsection{The $\pi / 2-\pi / 2$ interferometer}

For pedagogical purposes, let us first consider the simplest configuration described in Figure2 a. We start from an initial single-atom wave plane of momentum $\mathbf{p}$ in the internal state $|g\rangle$. This state is coupled to the plane wave of momentum $\mathbf{p}+\hbar \mathbf{k}_{1}-\hbar \mathbf{k}_{2}$ via a Raman transition. This transition is induced by two counterpropagating laser beams $\left(\mathbf{k}_{1}, \omega_{1}\right)$ and $\left(\mathbf{k}_{2}, \omega_{2}\right)$. We denote by $\mathbf{v}_{g}$ the velocity of the atoms in state $|g\rangle$ and $\mathbf{v}_{e}=\mathbf{v}_{g}+\hbar\left(\mathbf{k}_{1}-\mathbf{k}_{2}\right) / m$ the velocity of those in state $|e\rangle$. We apply a sequence of two $\pi / 2$ pulses. After the first pulse (at time $t_{1}$ ), the atoms are in a coherent superposition: $|\psi\rangle=\left(|g\rangle-i e^{-i \phi\left(t_{1}\right)}|e\rangle\right) / \sqrt{2}$. During a time $T_{R}$ of free propagation, each state accumulates a phase that depends both on its internal state and its kinetic energy:

$$
|\psi\rangle=\frac{1}{\sqrt{2}}\left(e^{-i \phi_{g}}|g\rangle-i e^{-i\left(\phi_{e}+\phi\left(t_{1}\right)\right)}|e\rangle\right)
$$

where:

$$
\begin{aligned}
\phi_{g} & =\frac{1}{2} m \mathbf{v}_{g}^{2} \times \frac{T_{\mathrm{R}}}{\hbar} \\
\phi_{e} & =\frac{1}{2} m \mathbf{v}_{e}^{2} \times \frac{T_{\mathrm{R}}}{\hbar}
\end{aligned}
$$

After the second $\pi / 2$ pulse (at time $t_{2}$ ) the atom is in the following state:

$$
|\psi\rangle=\frac{1}{2}\left[\left(e^{-i \phi_{g}}-e^{-i\left(\phi_{e}+\phi\left(t_{1}\right)-\phi\left(t_{2}\right)\right.}\right)|g\rangle-i\left(e^{-i\left(\phi_{g}+\phi\left(t_{2}\right)\right)}+e^{-i\left(\phi_{e}+\phi\left(t_{1}\right)\right)}\right)|e\rangle\right] .
$$

The probability to find the atom in the state $|e\rangle$ is then:

$$
|\langle e \mid \psi\rangle|^{2}=\frac{1}{2}(1+\cos \Phi)
$$

where the phase $\Phi$ is:

$$
\begin{aligned}
\Phi=\phi_{e}-\phi_{g}+\phi\left(t_{1}\right)-\phi\left(t_{2}\right) & =\delta \times T_{\mathrm{R}}+\left(\frac{1}{2} m \mathbf{v}_{e}^{2}-\frac{1}{2} m \mathbf{v}_{g}^{2}\right) \times \frac{T_{\mathrm{R}}}{\hbar} \\
& =\delta \times T_{\mathrm{R}}+\left(\frac{\mathbf{v}_{e}+\mathbf{v}_{g}}{2}\right) \cdot\left(\mathbf{v}_{e}-\mathbf{v}_{g}\right) \frac{m T_{\mathrm{R}}}{\hbar}
\end{aligned}
$$

The key point is that the phase depends both on the mean velocity and on the velocity difference between the two trajectories. In the case of counter-propagating Raman transitions, the difference in velocity is well known and equal to $\hbar\left(k_{1}+k_{2}\right) / m$. The interesting quantity is then the mean velocity of the atoms. The interferometer implemented by two $\pi / 2$ light pulses is 
sensitive to the initial velocity of atoms. Consequently, the fringe position will depend on the velocity of the atoms. This means that one can use this kind of interferometer only in the case where the initial velocity is well known. This was the case, for example, in the experiment of Pritchard and co-workers [17. There, they started from a Bose Einstein condensate at rest (see Perrin's paper in this issue). Because the initial velocity distribution was subrecoil $\left(\Delta v \ll v_{r}\right)$, they could assume that $v_{g}=0$, and write the phase shift as: $\Phi=\delta T_{\mathrm{R}}+2 \hbar k^{2} T_{\mathrm{R}} / m$. In their experiment they were able to measure the recoil energy and therefore the influence of the refractive index on the recoil momentum. However, in the case of a wide initial velocity distribution, the fringes will wash out when one averages over this distribution. This effect should of course be avoided for high-precision measurement but is used in some experiments to probe the initial velocity distribution and coherence properties of gas [18. For example, if one starts with a thermal Maxwell-Boltzmann distribution (velocity distribution in $e^{-m v^{2} /(2 k T)}$ ), the phase $\Phi$ has a gaussian distribution with a variance $\sigma_{\boldsymbol{\Phi}}$. Given that the average value of $\cos \Phi$ is equal to $e^{-\frac{\sigma_{\Phi}^{2}}{2}}$, this reduces the contrast of the fringes pattern by a factor of $\exp \left[-\pi\left(v_{r} T_{\mathrm{R}} / \lambda_{\mathrm{th}}\right)^{2}\right]$ where $\lambda_{\mathrm{th}}=\sqrt{2 \pi \hbar^{2} / m k T}$ is the de Broglie wavelength of an atom of thermal energy $k T$ ( $k$ is Boltzmann constant). This reduction of the contrast corresponds to the first-order correlation function of a thermal gas for a distance equal to the separation distance accumulated between the two $\pi / 2$ pulses $\left(2 v_{r} T_{R}\right)$.

\subsection{Interferometer with four $\pi / 2$ pulses}

To take full advantage of atom interferometry for high-precision measurements, one should choose a configuration where the phase shift between the two paths is independent of the initial velocity. This can be achieved if the interferometer is closed i.e. two classical trajectories (the center of mass trajectories) starting from the same initial point with initial velocity inducing the two paths of the interferometer end up at the same position (Figure. 2 $\mathrm{b}, 2 \mathrm{r}$ and $2 \mathrm{~d}$ but not 22a). To show this in a general case, let us call $v_{1,2}(t)$ the velocity of center of mass in the first and second arm of the interferometer. The general formula for the phase shift between the two paths due to the kinetic energy is:

$$
\frac{m}{\hbar} \int\left(\mathbf{v}_{1}(t)-\mathbf{v}_{2}(t)\right) \cdot\left(\frac{\mathbf{v}_{1}(t)+\mathbf{v}_{2}(t)}{2}\right) \mathrm{d} t
$$


The velocities $\mathbf{v}_{1}$ and $\mathbf{v}_{2}$ depend linearly on the initial velocity $v_{0}$, the phase term depending explicitly on $v_{0}$ is:

$$
\frac{m v_{0}}{2 \hbar}\left(\int \mathbf{v}_{1}(t) \mathrm{d} t-\int \mathbf{v}_{2}(t) \mathrm{d} t\right)
$$

This term equals zero if $\int \mathbf{v}_{1}(t) \mathrm{d} t=\int \mathbf{v}_{2}(t) \mathrm{d} t$, i.e. the two trajectories are closed.

An obvious scheme that satisfies this requirements is the $\pi / 2-\pi-\pi / 2$ sequence. In this case, the first $\pi / 2$ pulse acts as a beamsplitter. The two wave packets are separated by $\hbar\left(\mathbf{k}_{\mathbf{1}}-\mathbf{k}_{\mathbf{2}}\right)$ (assuming that each pulse drives Doppler-sensitive Raman transitions). After a time $T$, one applies a $\pi$ pulse that acts as mirror and redirects the interfering wave packets so that they overlap at the time $2 T$ of the second $\pi / 2$ pulse. Closed trajectories can be also obtained by using the sequence of two pair of $\pi / 2$ pulses. The direction of the Raman beams are reversed for the last pair in order to redirect the atoms. In the following we discuss the application of such sequences for the measurement either of the acceleration due to gravity or else the atomic recoil frequency.

\subsubsection{The $\pi / 2-\pi-\pi / 2$ scheme : the gravimeter}

The $\pi / 2-\pi-\pi / 2$ atom interferometer has been first demonstrated by Kasevich and Chu in 1992 [19] and used for gravimetry i.e. measurement of the acceleration due to gravity $g$. The light pulses are produced by two counter-counterpropagating vertical laser beams. Each pulse drives a Doppler-sensitive Raman transition between two atomic levels. In this experiment, Kasevich and Chu changed the frequency difference between the two Raman beams $\Delta \omega=\omega_{0}+\beta\left(t-t_{0}\right)$ in order to compensate the changing velocity of the falling atoms. If one uses the $\pi / 2-\pi-\pi / 2$ pulse sequence, the velocity difference $\mathbf{v}_{1}(t)-\mathbf{v}_{2}(t)$ due to the photon recoil is equal to $\hbar\left(\mathbf{k}_{1}-\mathbf{k}_{2}\right) / m$ before the $\pi$ pulse, and $-\hbar\left(\mathbf{k}_{1}-\mathbf{k}_{2}\right) / m$ after it. From eq. 15 we deduce the total phase :

$$
\Phi=\beta T_{\mathrm{R}}^{2}-\left(\mathbf{k}_{1}-\mathbf{k}_{2}\right) \cdot \mathbf{g} T_{\mathrm{R}}^{2}
$$

The population in the different internal states is measured scanning the parameter $\beta$. The value of $\beta$ that leads to $\Phi=0$ is given by:

$$
\beta=\left(\mathbf{k}_{1}-\mathbf{k}_{2}\right) \cdot \mathbf{g}
$$

In this case, there is no relative phase shift i.e. the changing Doppler shift is exactly canceled by the swept frequency difference of the Raman laser beams. This condition is insensitive to 
the time origin $t_{0}$ of the sweep and the delay $T_{R}$ between the pulses. Note that, in addition to the aforementioned phase shift, one should take into account the contribution of the initial net phase of the light beams $\phi_{i}^{0}$ at the time $t_{i}$ when they are switched on. This leads to an additional phase term $\phi_{1}^{0}-2 \phi_{2}^{0}+\phi_{3}^{0}[20$.

\subsubsection{The asymmetric Ramsey-Bordé interferometer}

Let us first consider the implementation described in Figure. 2 $\mathrm{d}$. This kind of interferometer is used to measure the recoil frequency. Here the directions of the Raman beams are reversed for the last two $\pi / 2$ pulses. Since the difference of the mean velocity between the first and second part is two recoils, eq. 15 yields to the following phase difference between the two arms of the interferometer:

$$
\Phi=2 \delta \times T_{\mathrm{R}}+4 \hbar k^{2} T_{\mathrm{R}} / m
$$

In a typical experiment, one varies the detuning $\delta$ in order to record the fringe pattern. The recoil frequency $\omega_{r}=\hbar k^{2} / 2 m$ is deduced from the central fringe. This scheme was first used by the Chu group to perform a measurement of recoil velocity [21/22].

\subsubsection{The symmetric Ramsey-Bordé interferometer}

This interferometer is a generalized version of the gravimeter and is depicted in Figure. 2rk. It involves a sequence of four $\pi / 2$ pulses without reversal of the direction of the Raman beams. We assume that the laser beams are oriented vertically . The delay between each pair of $\pi / 2$ pulses is $T_{R}$ while that between the first and third pulses is $T$. If we consider that the laser frequencies are swept so that:

$$
\begin{gathered}
\delta(t)=\delta_{0}+\gamma t \quad 0<t<T_{R} \\
\delta(t)=\delta_{1}+\gamma(t-T) \quad t>T
\end{gathered}
$$

the phase difference between the two arms is then given by

$$
\Phi=T_{\mathrm{R}}\left(\left(\delta_{1}-\delta_{0}\right)+(\boldsymbol{\Delta} \mathbf{v}+\mathbf{g} T) \cdot\left(\mathbf{k}_{1}-\mathbf{k}_{2}\right)\right)
$$

where the term $\boldsymbol{\Delta} \mathbf{v}$ allows for any velocity variation between the second and third pulses (on top of the trajectory). The phase is insensitive to the internal energy of the atoms. As discussed 
in the second part of this paper, we have used this scheme to measure the recoil velocity of atoms. The velocity variation is induced by the transfer of a large and well-defined number of photon momenta using a Bloch oscillation phenomena.

\subsection{Description in term of velocity selection}

There is an intuitive way of understanding the Ramsey-Bordé interferometer in terms of selection/measurement of a velocity distribution. This line of reasoning was adopted by Moler et al in their early paper on atom interferometry $[23]$.

In the usual Ramsey experiment (as performed for example in atomic clocks), the transition probability for a system driven with two $\pi / 2$ pulses separated by a delay $T_{\mathrm{R}}$ is a function

of the detuning $\delta$. This function is the product of the sinc function describing the resonance condition of each $\pi / 2$ pulse and a cosine function describing the interferences fringes, assuming that $\delta \ll \Omega$ (where $\Omega$ is the effective Rabi frequency):

$$
P(\delta)=\frac{\Omega^{2}}{\Omega^{2}+\delta^{2}} \sin ^{2}\left(\left(\sqrt{\Omega^{2}+\delta^{2}}\right) \tau\right) \cos ^{2}\left(\frac{\delta \times T_{R}}{2}\right)
$$

In the case of an atomic velocity sensor, because of the Doppler effect, the detuning depends on the velocity of each atom. This means that atoms transferred from state $|g\rangle$ to state $|e\rangle$ by the first two $\pi / 2$ pulses have a velocity distribution following a Ramsey pattern (velocity comb). The width of each "tooth" of this Ramsey pattern is proportional to $1 / T_{\mathrm{R}}$ while the position of the central fringe depends on the frequency of the lasers.

To measure the final velocity distribution, we use a second pair of $\pi / 2$ pulses that transfer atoms from $|e\rangle$ to $|g\rangle$ following a second Ramsey pattern in velocity space. The proportion of atoms transferred back to the initial state $|g\rangle$ depends on the overlaps of the two "velocity combs". As the frequency of the second pair of $\pi / 2$ pulses is tuned, this relative position varies and the probability for transferring atoms from $|e\rangle$ to $|g\rangle$ oscillates. The relative position of the central fringes indicates the Doppler effect that compensates any velocity change that occurs between selection and measurement. Furthermore, the longer the delay between the $\pi / 2$ pulses, the narrower is the width of the "teeth" of the Ramsey pattern and hence the resolution with which the frequency of the central peak can be located .

In the case of the symmetric Ramsey-Bordé interferometer, without gravity, if $\delta_{0}$ (resp. $\delta_{1}$ ) are the frequency for the first (resp. second) pair of $\pi / 2$ pulses, then the initial pattern is 
centered on the velocity $\mathbf{v}_{0}$ given by $\left(\mathbf{k}_{2}-\mathbf{k}_{1}\right) \cdot \mathbf{v}_{0}=\delta_{0}$. The value of $\delta_{1}$ corresponding to the central fringe is then $\delta_{0}+\left(\mathbf{k}_{2}-\mathbf{k}_{1}\right) \cdot \boldsymbol{\Delta} \mathbf{v}$. This result is identical to eq. 23

\section{Part II : A measurement of $h / m_{\mathrm{Rb}}$ combining atom interferometer and Bloch oscillations}

\section{Outline}

Quantum mechanics always links the mass $m$ of a particle to the Planck constant through the ratio $h / m$. Mass appears in the basic quantum mechanical equations of motion only in this ratio (in the Schrödinger equation, as well as in the relativistic equations of Dirac and KleinGordon). To compare quantum theories with experiment, only the measurement of this ratio is required and not $m$ independently. The most relevant example of this is that the ratio $h / m_{e}$ ( $m_{e}$ is the electron mass) is involved in the determination of the fine structure constant $\alpha$, via the Rydberg constant $R_{\infty}$ :

$$
\alpha^{2}=\frac{2 R_{\infty}}{c} \times \frac{h}{m_{e}}
$$

The ratio $h / m$ for an atomic system can be deduced from the measurement of the recoil velocity or energy combined with the accurate measurement of the photon wavelength $\lambda$. Since the preliminary measurement of the recoil splitting in saturation spectroscopy [24], laser cooling has stimulated renewed interest in such a determinations [22 25]. The aim of our experiment is to provide an improved value of the fine structure constant $\alpha$ from the measurement of the ratio $h / m$. The two quantities are related via [26]:

$$
\alpha^{2}=\frac{2 R_{\infty}}{c} \frac{A_{r}(X)}{A_{r}(e)} \frac{h}{m_{X}}
$$

where $R_{\infty}$ is the Rydberg constant, $A_{r}(e)$ is the relative atomic mass of the electron and $A_{r}(X)$ the relative mass of the particle $X$ with mass $m_{X}$. These factors are known with a relative uncertainty of $7 \times 10^{-12}$ for $R_{\infty}\left[2728,4.4 \times 10^{-10}\right.$ for $A_{r}(e)\left[29\right.$ and less than $2.0 \times 10^{-10}$ for $A_{r}(\mathrm{Cs})$ and $A_{r}(\mathrm{Rb})$ 30. Hence, the factor limiting the accuracy of $\alpha$ is the ratio $h / m_{X}$.

The fine structure constant is a corner stone of the adjustment of the fundamental physical constants 31|32. Its value is obtained from experiments in different domains of physics, such as the quantum Hall effect in solid state physics, or the measurement of the muonium ground-state hyperfine structure in atomic physics (see papers in this issue). At present, the most precise determinations of $\alpha$ have been deduced from the measurements of the electron anomaly $a_{e}$ 


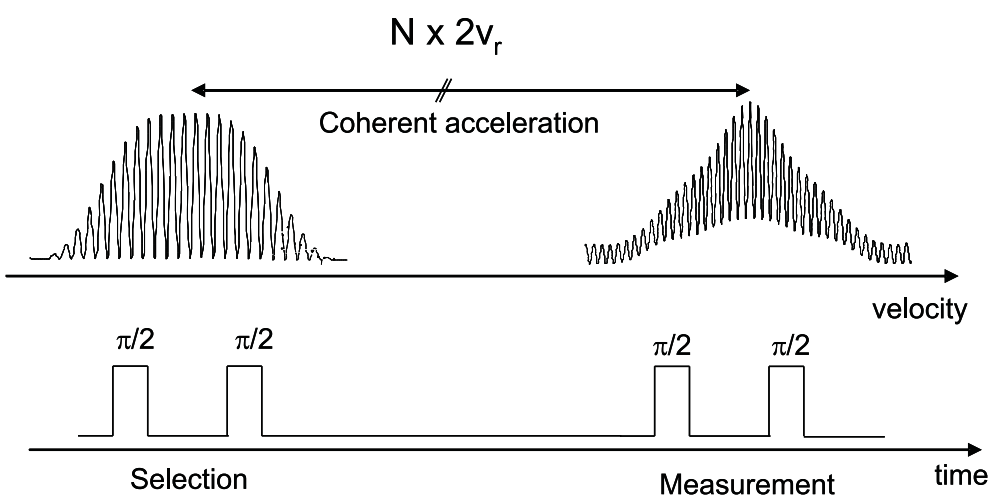

Fig. 3. We select a narrow velocity "comb" by a first pair of $\pi / 2$ Raman pulses (transition between the $F=2$ and $F=1$ hyperfine sublevels of the ground state). Then the atoms are accelerated by means of Bloch oscillations, and the final velocity of the atoms is probed with a second pair of $\pi / 2$-pulses.

made in the 1980's at the University of Washington [33] and, more recently, at Harvard 34 35. This last experiment and an impressive improvement of the quantum electrodynamics (QED) calculations [36 37] have led, in 2008, to a new determination of $\alpha$ with a relative uncertainty of $3.7 \times 10^{-10} 38$. Hence, the value of the fine structure constant recommended by the CODATA is mainly determined from $a_{e}$ measurement via QED calculation. This shows the need for other routes to the value of $\alpha$ independent of QED. The determination of $\alpha$ deduced from the measurements of $h / m_{\mathrm{Cs}} 22$ and $h / m_{\mathrm{Rb}}$ 25139] (where $m_{\mathrm{Cs}}$ and $m_{\mathrm{Rb}}$ are the masses of cesium and rubidium atoms) have an uncertainty of $7 \times 10^{-9}$ and $6.7 \times 10^{-9}$ respectively. Nowadays, the method based on the photon recoil measurement constitutes the unique alternative to increase our confidence in the recommended value of $\alpha$.

\section{Principle of the experiments}

In our work, the ratio $h / m$ is deduced from the measurement of the recoil velocity of a ${ }^{87} \mathrm{Rb}$ atom which absorbs or emits a photon, and the frequency of the photon involved. We use a symmetric Ramsey-Bordé interferometer (two pairs of $\pi / 2$ laser pulses). Each pulse drives a Doppler-sensitive Raman transition between the two hyperfine ground states $F=2$ and $F=1$. To explain the basic principle of the experiment, we describe our interferometer in momentum space (see Figure. 3): First we use a pair of $\pi / 2$ pulses to select a "velocity comb" in the internal state $F=1$ (corresponding to a Ramsey fringes pattern). The width of the envelope of this velocity comb varies inversely with the pulse duration $\tau$, while the fringe width varies as $1 / T_{R}$. We next transfer to these selected atoms a large number of photon momenta by means of Bloch oscillations as explained below. Finally, we use a second pair of $\pi / 2$ pulses to probe 


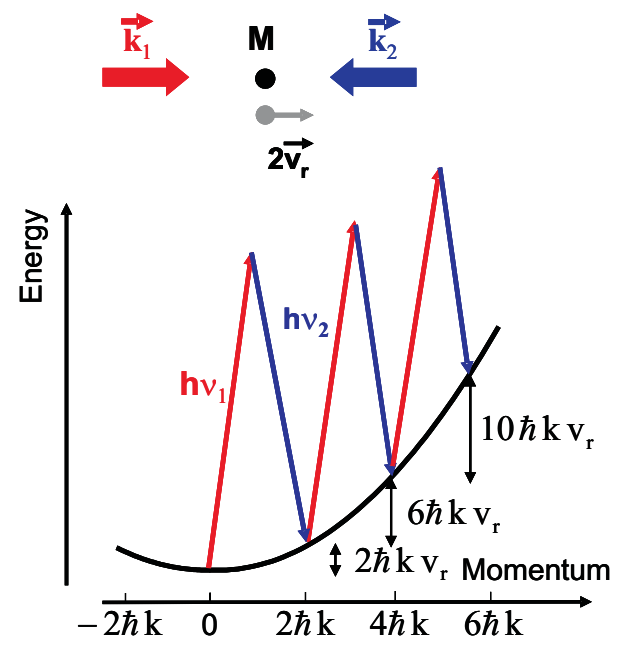

Fig. 4. Acceleration of cold atoms with a frequency chirped standing wave. The variation of energy versus momentum in the laboratory frame is given by a parabola. The energy of the atoms increases by the quantity $4(2 j+1) E_{r}$ at each cycle.

the accelerated velocity combs. By scanning the frequency of the last two Raman pulses, we perform the convolution of the initial velocity comb by the second one. The accuracy of the photon-recoil measurement depends on the number of recoils $(2 N)$ we are able to transfer to the atoms. Specifically, if we locate the center of the final velocity distribution (central fringe) with an accuracy of $\sigma_{\mathrm{v}}$, the accuracy of the recoil velocity measurement $\sigma_{\mathrm{v}_{\mathrm{r}}}$ is:

$$
\sigma_{\mathrm{v}_{\mathrm{r}}}=\frac{\sigma_{\mathrm{v}}}{2 N}
$$

\subsection{Bloch oscillation in an accelerated optical lattice}

Bloch oscillations were first observed in atomic physics a decade ago, by groups in Paris [0]41] and in Austin [42]. Similar effects have also been mentioned by Bordé [43. In a simple way, they can be viewed as two-photon transitions in which the atom begins and ends in the same energy level, so that its internal state $(F=1)$ remains unchanged while its velocity has increased by $2 v_{r}$ per oscillation (see Figure. 4). Bloch oscillations are produced in a one-dimensional optical lattice whose wells are accelerated as the relative frequency of two counter-propagating laser beams is swept linearly. The frequency difference $\Delta \nu$ is increased so that, because of the Doppler effect, the beams are periodically resonant with the same atoms $\left(\Delta \nu=4(2 j+1) E_{r} / h\right.$, $j=0,1,2,3$. where $E_{r} / h$ is the recoil energy in frequency units and $j$ the number of transitions). This leads to a succession of rapid adiabatic passages of the atoms between momentum states differing by $2 h \nu / c$. In the solid-state physics approach, this phenomenon is known as Bloch 
oscillations in the fundamental energy band of a periodic optical potential. In an optical lattice, the atoms are subjected to a constant inertial force obtained by the introduction of the tunable frequency difference $\Delta \nu$ between the two waves that create the optical potential [40]. The usefulness of the Bloch oscillations technique is that it allows one to transfer a large number of photon momenta (efficiency of $99.97 \%$ per recoil) to any velocity shape that lies within the first Brillouin zone (defined by $[-\hbar k,+\hbar k]$ in momentum space), in a coherent way, i.e. without any change of the velocity shape 44,39].

Before closing this section, we mention an elegant configuration for Bloch oscillations, which occurs when an atom is placed in a vertical standing wave. The atom, initially at rest, starts to fall because of gravity. When its momentum has reached the value $-\hbar k$, it absorbs a photon from the up-propagating wave and emits another one in the down-propagating wave. At the end of this $\Lambda$ transition, its momentum is equal to $+\hbar k$. The atomic momentum thus oscillates between $+\hbar k$ and $-\hbar k$. The time required for this oscillation is equal to $T=2 h \nu / c M g$ where $g$ is the acceleration due to gravity, $\nu$ the optical frequency of the wave and $c$ the speed of the light. In the case of rubidium atom, the oscillation frequency is about $830 \mathrm{~Hz}$. This effect has been observed by our group [45] and briefly described in previous papers [46]47]. It has also been reported by other groups [484950].

\section{Experimental setup}

Our experimental setup (Figure. 5) has been previously described in detail elsewhere [39]. Briefly, ${ }^{87} \mathrm{Rb}$ atoms are captured from a background vapor in a $\sigma^{+}-\sigma^{-}$configuration magnetooptical trap (MOT). The trapping magnetic field is then switched off and the atoms are cooled to about $3 \mu K$ in an optical molasses. After the cooling process, we apply a bias field of $10 \mu \mathrm{T}$. The atoms are then optically pumped into the $F=2, m_{F}=0$ ground state. We apply the first pair of $\pi / 2$ pulses which select a comb of velocities. The $\pi / 2$ pulses are produced by two vertical counter-propagating laser beams (Raman beams). The selection is implemented by transferring the resonant atoms from $5 S_{1 / 2},\left|F=2, m_{F}=0\right\rangle$ state to $5 S_{1 / 2},\left|F=1, m_{F}=0\right\rangle$ state and pushing away the atoms remaining in the state $F=2$. To push away the unwanted atoms, we apply after the first $\pi / 2-\pi / 2$-pulse sequence, a laser beam resonant with the $5 S_{1 / 2}(F=2)$ to $5 P_{3 / 2}(F=3)$ cycling transition. Atoms in the state $F=1$ make $N$ Bloch oscillations in an accelerated vertical optical lattice.

We then perform the velocity measurement step using the second pair of Raman $\pi / 2$-pulses, whose frequency is $\delta_{\text {meas }}$. The populations in the levels $F=1$ and $F=2$ are measured separately 


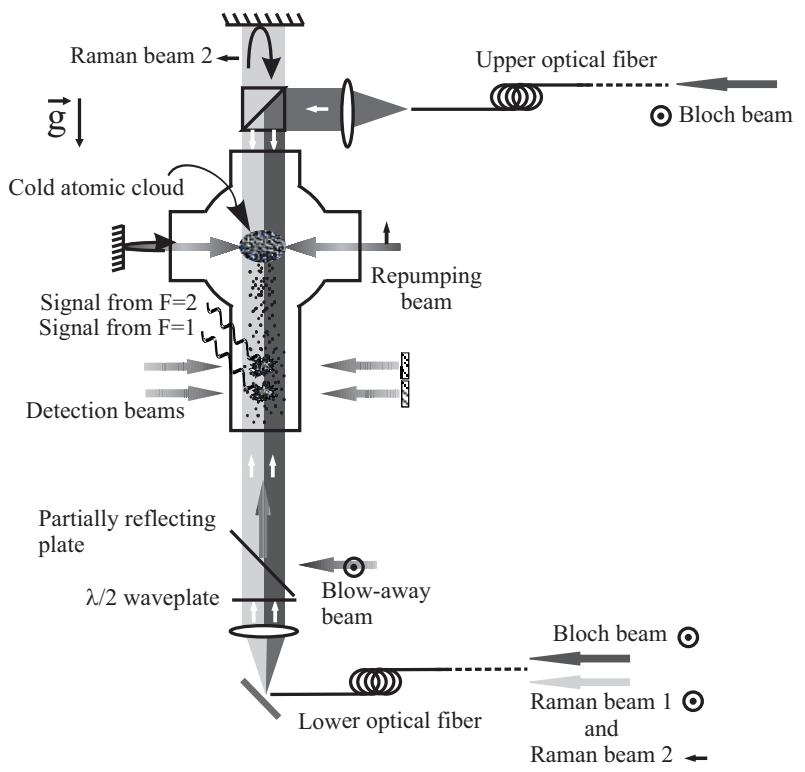

Fig. 5. Scheme of the experimental setup: the cold atomic cloud is produced in a MOT (the cooling laser beams are not shown). The Raman and the Bloch beams are vertical. The Raman beams and the upward Bloch beam are injected into the same optical fiber. The "blow-away" beam is tuned to the one photon transition and allows us to clear the atoms remaining in $F=2$ after the selection step. The populations in the hyperfine levels $F=1$ and $F=2$ are detected by fluorescence $15 \mathrm{~cm}$ below the MOT using a time of flight technique.

by using the one-dimensional time of flight technique. To plot the final velocity distribution we repeat this procedure by scanning the Raman beam frequency $\delta_{\text {meas }}$ of the second pulse.

The Raman beams are produced by two frequency-stabilized diode lasers. Their beat frequency is precisely controlled by a frequency chain allowing one to switch easily from the selection frequency $\left(\delta_{\text {sel }}\right)$ to the measurement frequency $\left(\delta_{\text {meas }}\right)$. One of the lasers is locked to a peak of a highly stable Fabry-Perot cavity. The two Raman beams have linear orthogonal polarizations and are coupled into the same polarization-maintaining optical fiber. The pair of Raman beams is sent through the vacuum cell. The counter-propagating configuration is achieved using a polarizing beam-splitter cube and a horizontal retro-reflecting mirror placed above the exit window of the cell.

The standing wave used to create the 1-D optical lattice is generated by a Ti:Sapphire laser, whose frequency is stabilized to the same highly stable Fabry-Perot cavity used for the Raman beam laser diodes. Its frequency is continuously measured by counting the beatnote with another frequency stabilized Ti-Sapphire laser referenced to a Rb two-photon standard 5152

The Ti:Sapphire laser beam is split into two parts. To implement the timing sequence, we use several acousto-optic modulators to control the intensity and frequency of different laser 
beams. The optical lattice is blue detuned by $\sim 40 \mathrm{GHz}$ from the $5 S_{1 / 2}-5 P_{3 / 2}$ single photon transition. The power of the lattice beams is raised slowly $(500 \mu \mathrm{s})$ in order to load all the atoms adiabatically into the first Bloch band. To perform the coherent acceleration, the frequency difference of the two laser beams generating the optical lattice is swept linearly. Then, the lattice intensity is slowly lowered in $500 \mu$ s to bring atoms back into a well-defined momentum state. The optical potential depth is $\sim 100 E_{r}$. With these lattice parameters, the spontaneous emission is negligible. For an acceleration of about $2000 \mathrm{~ms}^{-2}$ we transfer about 1200 photon momenta in $3 \mathrm{~ms}$. To avoid atoms from reaching the upper window of the vacuum chamber, we use a double acceleration scheme: instead of selecting atoms at rest, we first accelerate them using Bloch oscillations and then we perform the three-step sequence: selection-accelerationmeasurement. In this way, the atomic velocity at the measurement step is close to zero. In the vertical direction, an accurate determination of the recoil velocity would require an accurate measurement of the acceleration due to gravity $g$. In order to circumvent this difficulty, we make a differential measurement by accelerating the atoms in opposite directions (upward and downward trajectories) keeping the same delay between the two pairs $\pi / 2$-pulses. Thus the photon-recoil measurement is a determination of the frequency difference between the central fringes of two opposite interferometers (upward and downward) illustrated in Figure. 6 .

The ratio $\hbar / m$ can then be deduced from the formula:

$$
\frac{\hbar}{m}=\frac{\left(\delta_{\text {sel }}-\delta_{\text {meas }}\right)^{\text {up }}-\left(\delta_{\text {sel }}-\delta_{\text {meas }}\right)^{\text {down }}}{2\left(N^{\text {up }}+N^{\text {down }}\right) k_{B}\left(k_{1}+k_{2}\right)}
$$

where $\left(\delta_{\text {meas }}-\delta_{\text {sel }}\right)^{\text {up/down }}$ corresponds respectively to the center of the final velocity distribution for the up and the down trajectories, $N^{\text {up/down }}$ are the number of Bloch oscillations in both opposite directions, $k_{B}$ is the Bloch wave vector and $k_{1}$ and $k_{2}$ are the wave vectors of the Raman beams.

Moreover, the contribution of some systematic effects (energy level shifts) to $\delta_{\text {sel }}$ and $\delta_{\text {meas }}$ changes sign when the directions of the Raman beams are interchanged. To improve the experimental protocol, for each up or down trajectory, the directions Raman beams are reversed and we record two velocity spectra. Finally, each determination of $h / m_{\mathrm{Rb}}$ and $\alpha$ is obtained from four velocity spectra. 


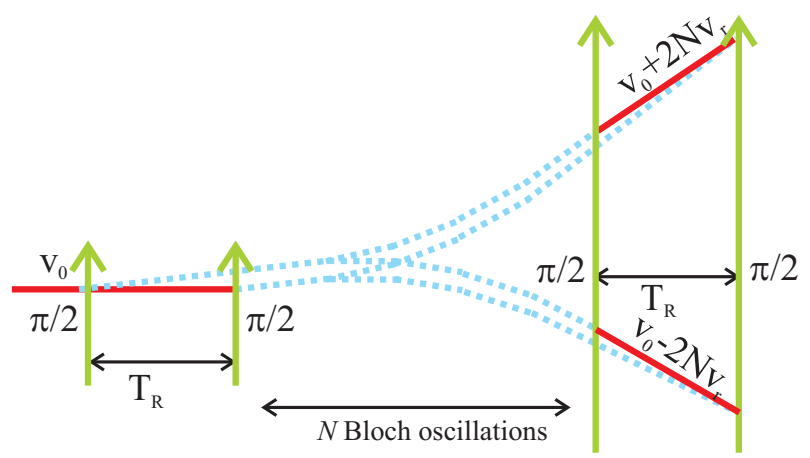

Fig. 6. Scheme of the interferometers used for the measurement of $h / m_{\mathrm{Rb}}$. The first pair of $\pi / 2$ pulses selects a comb of velocities which is measured by the second pair of $\pi / 2$ pulses. Between these two pairs of pulses, the atoms are accelerated upwards or downwards. The solid line corresponds to the atom in the $F=2$ level, and the dashed line to the $F=1$ level.
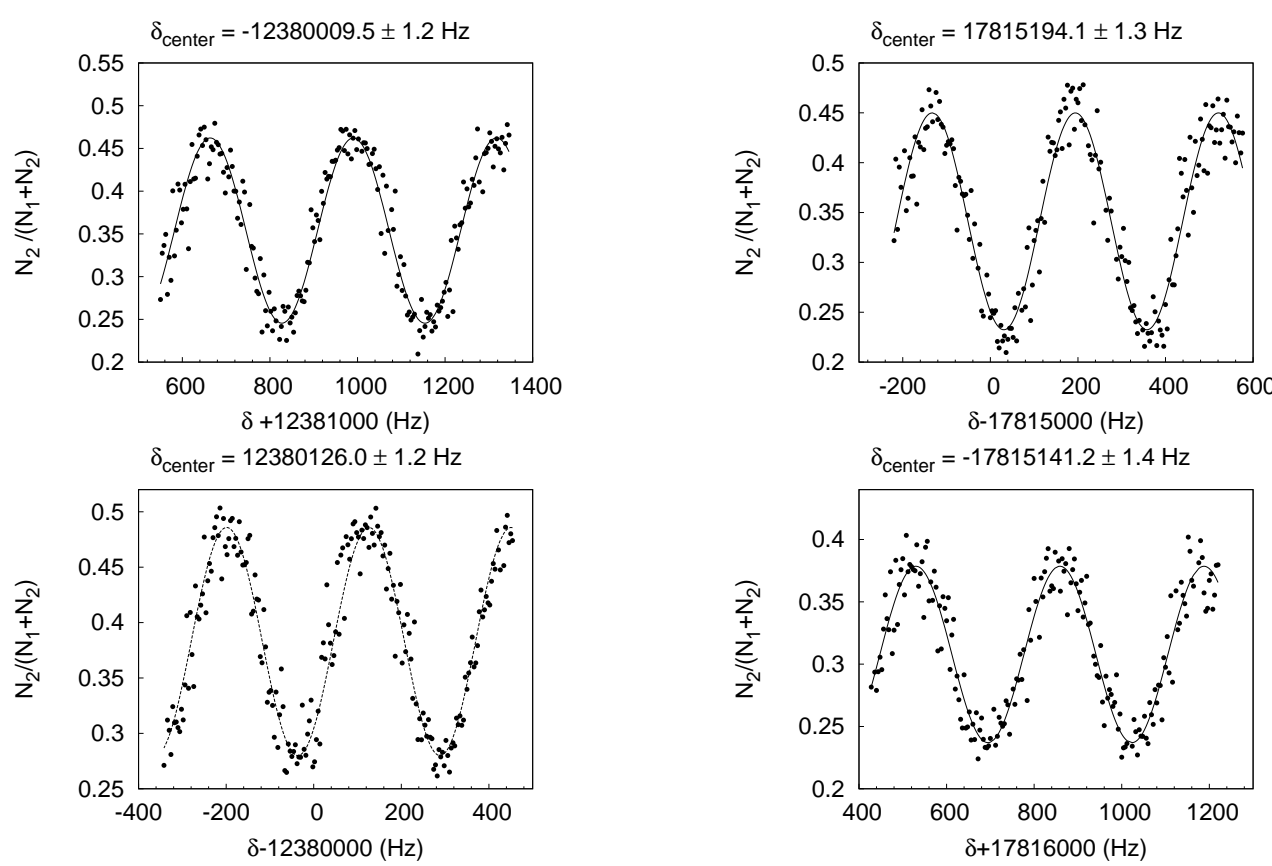

Fig. 7. Spectra obtained by atomic interferometry which show the proportion of atoms in the $F=2$ level $\left(N_{2} /\left(N_{1}+N_{2}\right)\right)$ in function of the frequency difference $\delta_{\text {meas }}-\delta_{\text {sel }}$ in hertz. The two spectra on the left corresponds to the upwards acceleration (400 Bloch oscillations) and the two spectra on the right to the downwards acceleration (600 Bloch oscillations).

\section{Preliminary results}

In this section we present our recent and preliminary determination of the fine structure constant. The measurement of the ratio $h / m_{\mathrm{Rb}}$ is obtained by combining a Ramsey-Bordé interferometer with Bloch oscillations in an accelerated lattice (see the experimental protocol described in Figure. 6). A typical set of four spectra used to provide one determination of the ratio $h / m_{\mathrm{Rb}}$ is shown in Figure. 7 In this case, the total number of Bloch oscillations is $N^{\text {up }}+N^{\text {down }}=1000$, corresponding to 2000 recoil velocities between the up and down trajectories. The duration of 


\begin{tabular}{lcc}
\hline Source & $\begin{array}{c}\text { Correction } \\
\left(\times 10^{-9}\right)\end{array}$ & $\begin{array}{c}\text { Relative uncertainty } \\
\left(\times 10^{-9}\right)\end{array}$ \\
\hline Laser frequencies & -2 & 0.4 \\
Beams alignment & -11.9 & 2 \\
Wavefront curvature and Gouy phase & 7 & 2.5 \\
2nd order Zeeman effect & -1.45 & 1 \\
Quadratic magnetic force & -0.18 & 0.2 \\
Gravity gradient & 0 & 0.02 \\
light shift (one photon transition) & 0 & 0.1 \\
light shift (two photon transition) & 0.58 & 0.01 \\
light shift (Bloch oscillation) & 0 & 0.2 \\
Index of refraction atomic cloud & -0.41 & 0.3 \\
Index of refraction background vapor & -8.36 & 0.3 \\
\hline \hline Global systematic effects & 3.4 \\
\hline
\end{tabular}

Table 1. Error budget on the determination of $\alpha$ (Systematic effects and relative uncertainty).

each $\pi / 2$ pulse is $400 \mu$ s and the time $T_{R}$ is $2.6 \mathrm{~ms}$ (the total time for a pair of $\pi / 2$ pulses is $3.4 \mathrm{~ms})$. The central fringe is determined with an uncertainty lower than $1.4 \mathrm{~Hz}\left(\sim v_{r} / 10000\right)$. Each spectrum is plotted with 200 points and is obtained in 6 min. We obtain an excellent fringes visibility of about $30 \%$ for 600 Bloch oscillations. The analysis of 125 determinations of $\alpha$ leads to the statistical uncertainty of $3.3 \times 10^{-9}$ with $\chi^{2} /(n-1)=1.55$.

The systematic effects taken into account to determine the value of the fine structure constant are summarized in Table 1] These effects are detailed in a previous paper [39. Up to now, the main contributions come from the corrections due to the wavefront curvature and the second-order Zeeman effect due to the residual magnetic field. This last correction is determined by carefully mapping the magnetic field in the interaction area. It should be reduced by implementing a magnetic shielding. The total relative uncertainty on $\alpha$ is then $4.7 \times 10^{-9}$.

The preliminary value of the fine structure constant is:

$$
\alpha^{-1}=137.03599887(64) \quad\left[4.7 \times 10^{-9}\right]
$$

This value is in good agreement with our last determination $\alpha[06]$ published in 2006 [39]. It was obtained by using an non-interferometric method based on the $\pi-\pi$ Raman pulses for the velocity sensor.

$$
\alpha^{-1}[06]=137.03599884(91) \quad\left[6.7 \times 10^{-9}\right]
$$

Complementary measurements are in progress in order to determine the final value of the fine structure constant based on this interferometric approach. 


\section{Conclusion}

In this lecture we have given a brief analysis of atom interferometers based on the arrangements of $\pi / 2$ and $\pi$ laser pulses. We have discussed their applications to the measurement of the gravitational acceleration and atomic recoil frequency. We have described in detail an experimental method which combines the Bloch oscillation process with a Ramsey-Bordé interferometer. We believe that this combination is a very promising method for improving the accuracy of atomic recoil measurements.

An alternative way to improve the sensitivity of atom interferometer consists in using a large momentum beam-splitter. Such beam-splitters can be implemented using multi-photon Bragg diffraction in a static optical lattice 35315455] or Bloch oscillations in a moving lattice [56. In this case, the separation between the interfering atomic wavepackets is performed by $2 N \hbar k$ instead $2 \hbar k$ for a $\pi / 2$ light pulse (where $N$ is the number of photon momenta imparted to the atoms as the result of the interaction with the optical lattice). In this way, one increases the area of the interferometer and thus its sensitivity. These investigations are in progress [57] and should lead to a growing number of applications of atom interferometry, metrology and applied physics.

This experiment is supported in part by the Laboratoire National de Métrologie et d'Essais (Ex. Bureau National de Métrologie)(Contrat 033006), by IFRAF (Institut Francilien de Recherches sur les Atomes Froids) and by the Agence Nationale pour la Recherche, FISCOM Project-(ANR06-BLAN-0192).

\section{References}

1. P.R. Berman, ed. 1997, Atom Interferometry, (New York: Academic Press).

2. P.L. Gould, G. A. Ruff and D.E. Pritchard. Phys. Rev. Lett. 56, 827 (1986).

3. C.J. Bordé, Phys. Lett. A 140, 10 (1989).

4. F. Riehle, T. Kisters, A. Witte, J. Helmcke and C. Bordé, Phys.Rev. Lett. 67, 177 (1991).

5. M. Kasevich and S. Chu, Phys.Rev. Lett. 67, 181 (1991).

6. U. Sterr, K. Sengstock, J. H. Muller, D. Bettermann, and W. Ertmer, Appl. Phys. B 54, 341 (1992).

7. M. Jacquey, M. Büchner, G. Trénec and J. Vigué, Phys. Rev. Lett. 98240405 (2007).

8. D. W. Keith, M. L. Shattenburg, H. I. Smith and D.E. Pritchard Phys. Rev. Lett. 61, 1580 (1988).

9. O. Carnal and J. Mlynek, Phys.Rev. Lett. 66, 2689 (1991). 
10. F. Shimizu, K. Shimizu and H. Takuma, Phys. Rev. A 4646 (1992).

11. M. Kasevich, D.S. Weiss, E. Reiis, K. Moler, S. Kasapo and S. Chu, Phys. Rev. Lett. 66, 2297 (1991).

12. Y.V. Baklanov, B. Y. Dubetsky and V.P. Chebotayev, Appl. Phys. 9, 171 (1976).

13. J.C. Berquist, S.A. Lee and J. L. Hall, Phys. Rev. Lett. 38, 159 (1977).

14. Ch. J. Bordé, S. Avrillier, Ch. Salomon, A. Van Lerberghe, Ch. Bréant, D. Bassi and G. Scoles, Phys. Rev. A 30, 1836-1848 (1984).

15. C. J. Bordé, In Laser Spectroscopy X (Edited by M. Ducloy, E. Giacobino and G. Camy), Singapore, World Scientific, 1992, 239-245.

16. E.P. Storey and C. Cohen-Tannoudji., J. Phys. II France, 4, 1999-2027 (1994).

17. G. K. Campbell, A. E. Leanhardt, J. Mun, M. Boyd, E. W. Streed, W. Ketterle and D. E. Pritchard, Phys. Rev. Lett. 94, (2005) 170403.

18. M. Hugbart, J.A. Retter, F. Gerbier, A.F. Varón, S. Richard, J.H. Thywissen, D. Clément, P. Bouyer and A. Aspect, Eur. Phys. J. D 35, 155-163 (2005).

19. M. Kasevich and S. Chu Appl. Phys. B 54, 321 (1992).

20. A. Peters, K. Y. Chung, and S. Chu, Metrologia 38, 25 (2001).

21. D. S. Weiss, B. C. Young, and S. Chu, Appl. Phys. B 59, 217 (1994).

22. A. Wicht, J.M. Hensley, E. Sarajilic and S. Chu, Physica Scripta T102, (2002) 82.

23. K. Moler and D. S. Weiss, M. Kasevich and S. Chu, Phys. Rev. A 45, 344 (1992).

24. J.L.Hall, Ch.J.Bord, K.Uehara, Phys. Rev. Lett. 37,1339 (1976).

25. P. Cladé, E. de Mirandes, M. Cadoret, S. Guellati-Khélifa, C. Schwob, F. Nez, L. Julien and F. Biraben, Phys. Rev. Lett. 96, 033001-1 (2006).

26. B.N. Taylor, Metrologia 31, (1994)181.

27. Th. Udem, A. Huber, B. Gross, J. Reichert, M. Prevedelli, M. Weitz and T.W. Hänsch, Phys. Rev. Lett. 79, (1997) 2646.

28. C. Schwob, L. Jozefowski, B. de Beauvoir, L. Hilico, F. Nez, L. Julien, F. Biraben, O. Acef and A. Clairon, Phys. Rev. Lett. 82, (1999) 4960.

29. T. Beir, H. Häffner, N. Hermansphan, S.G. Karshenboim, H.-J. Kluge, W. Quint, S. Sthal, J. Verdú and G. Werth, Phys. Rev. Lett. 88, (2002) 011603.

30. M.P. Bradley, J.V. Porto, S. Rainville, J.K. Thompson and D.E. Pritchard, Phys. Rev. Lett.83, (1999) 4510.

31. P. Mohr and B.N. Taylor, Rev. Mod. Phys. 77, (2005) 1.

32. P. J. Mohr, B.N. Taylor and D. B. Newell Rev. Mod. Phys. 80, 633 (2008).

33. R.S. Van Dyck, P.B. Schwinberg and H.G. Dehmelt, Phys. Rev. Lett. 59, (1987) 26.

34. G. Gabrielse, D. Hanneke, T. Kinoshita, M. Nio and B. Odom, Phys. Rev. Lett. 97, (2006) 030802.

35. B. Odom, D. Hanneke, B. D’Urso and G. Gabrielse, Phys. Rev. Lett. 97, (2006) 030801. 
36. T. Kinoshita and M. Nio, Phys. Rev. D 7, (2006) 013003.

37. G. Gabrielse, D. Hanneke, T. Kinoshita, M. Nio and B. Odom, Phys. Rev. Lett. 99, (2007) 039902.

38. D. Hanneke, S. Fogwell, and G. Gabrielse, Phys. Rev. Lett. 100, 120801 (2008).

39. P. Cladé, E. de Mirandes, M. Cadoret, S. Guellati-Khélifa, C. Schwob, F. Nez, L. Julien and F. Biraben, Phys. Rev. A 102, (2006) 052109.

40. M. Ben Dahan, E. Peik, J. Reichel, Y. Castin and C. Salomon, Phys. Rev. Lett. 76, (1996) 4508.

41. E. Peik, M. Ben Dahan, I. Bouchoule, Y. Castin and C. Salomon, Phys. Rev. A 55, (1997) 2989.

42. S. R. Wilkinson, C. F. Bharucha, K. W. Madison, Qian Niu, and M. G. Raizen, Phys. Rev. Lett. 76, (1996) 4512 .

43. C. Bordé, In frequency standards and Metrology (A. De Marchi, ed.), Springer-Verlag,1989.

44. R. Battesti, P. Cladé, S. Guellati-Khélifa, C. Schwob, B. Grémaud, F. Nez, L. Julien and F. Biraben, Phys. Rev. Lett. 92, (2004) 253001-1.

45. P. Cladé, S. Guellati-Khélifa, C. Schwob, F. Nez, L. Julien and F. Biraben, Europhys. Lett., 71, (2005) 730 .

46. C. Schwob, L. Masson, B. Grmaud, S. Guellati, F. Nez, L. Julien and F. Biraben J. Phys. IV France 10 (2000) Pr8-153.

47. R. Battesti, P. Clad, S. Guellati-Khlifa, C. Schwob, B. Grmaud, F. Nez, L. Julien and F. Biraben, J. Opt. B : Quantum Semiclass. Opt. 5 (2003) pp S178-S182.

48. G. Roati, E. de Mirandes, F. Ferlaino, H. Ott, G. Modugno and M. Inguscio, Phys. Rev. Lett. 92, (2004) 230402.

49. G. Ferrari, N. Poli, F. Sorrentino and G.M. Tino GM, Phys. Rev. Lett. 97, (2006) 060402.

50. M. Gustavsson, E. Haller, M.J. Mark, J.G. Danzl, G. Rojas-Kopeinig and H.C. Naegerl, Phys. Rev. Lett. 100, (2008) 080404.

51. D. Touahri, O. Acef, A. Clairon, J.-J. Zondy, R. Felder, L. Hilico, B. de Beauvoir, F. Biraben and F. Nez, Opt. Commun. 133, (1997) 471.

52. B. de Beauvoir, C. Schwob, O. Acef, L. Jozefowski, L. Hilico, F. Nez, L. Julien, A. Clairon, and F. Biraben Eur. Phys. J. D 12 (2000) 61-93.

53. D. M. Giltner, R. W. McGowan, and S.A. Lee, Phys. Rev. Lett. 75, 2638 - 2641 (1995).

54. S. Gupta, K. Dieckmann, Z. Hadzibabic, and D. E. Pritchard, Phys. Rev. Lett. 89, 140401 (2002).

55. A. E. A. Koolen, G. T. Jansen, K. F. E. M. Domen, H. C. W. Beijerinck, and K. A. H. van Leeuwen, Phys. Rev. A 65, 041601 (2002).

56. J. H. Denschlag, J.E. Simsarian, H. Häffner, C. McKenzie, A. Browaeys, D. Cho, K. Helmerson, S.L. Rolston, and W.D. Phillips, J. Phys. B: At. Mol. Opt. Phys. 35 (2002) 3095-3110.

57. H. Muller, S. W. Chiow, S. Herrmann and S. Chu, AIP Conf. Proc. 977 (2008) 291. 